\title{
Diagnostico de Estrategia de Innovación en Grupos de Investigación
}

\author{
JhonWilder Zartha Sossa', Gina Lía Orozco Mendoza², Jorge IvánVergara Sornozaª, Diego Jessie Martínez
}

\begin{abstract}
This study involves a statistical analysis based on the results of the diagnostic strategy in innovation in 52 research groups at one university in Medellin - Colombia, to validate the currently situation of the groups towards innovation, strategies, structures of development equipments among others. The methodology was designed by the Policy and Technology Management Research Group, based on different models such as, methodology in technological management of projects, diagnosis of innovation by Albacete, Cataluña and methodology for innovation strategy by Melissa Schilling. The results offer basis for the formulation and implementation of innovative strategies in the different groups allowing the improvement in the innovation generation of products, services, processes, markets as well as to improve its competitiveness.
\end{abstract}

Keywords: Innovation; research groups; innovation strategy.

\section{Resumen}

Este estudio comprende un análisis estadístico sobre los resultados del diagnóstico de estrategia de innovación en 52 grupos de investigación de una Universidad en Medellín - Colombia, para validar el estado actual de los grupos frente a la innovación, sus estrategias, estructuras de equipos de desarrollo y otros temas relevantes. La metodología fue diseñada por el Grupo de Investigación de Política y Gestión Tecnológica, fundamentada en diferentes modelos: Metodología de Gestión Tecnológica por proyectos, diagnósticos de innovación de Albacete, Cataluña y metodología de estrategia de innovación de Melissa Schilling. Los resultados ofrecen una base para la formulación e implementación de estrategias de innovación en grupos que permitan mejorar la generación de innovación en productos, servicios, procesos, mercados y mejorar su competitividad.

Palabras claves: Innovación; grupos de investigación; estrategia de innovación.

'Grupo política y Gestión Tecnológica, Universidad Pontificia Bolivariana, Circular I No. 70-0I, Bloque II - I05, Medellín, Colombia, 05003I, +57(4) 3544522, Email: jhon.zartha@upb.edu.co

${ }^{2}$ Circular I No. 70-0I, Bloque II - I05, Medellín, Colombia, 05003 I, +57(4) 3544522, Email: gina.orozco@upb.edu.co

${ }^{3}$ Circular 57216 apto 203, Laureles. Medellín, Colombia, 3013562724, Email: jorgesornoza@yahoo.com

${ }^{4}$ Cra 37A \# 8-43 Of.802 Medellín, Colombia,Tel. +57 42524088 


\section{Introducción}

La Gestión de la Tecnología y la Innovación se han convertido en los constituyentes claves en el entorno empresarial para garantizar el crecimiento (Martínez, 2009), la permanencia en el tiempo, enfrentarse a las situaciones cambiantes actuales y de futuro, generar avance tecnológico (Merrifield, 2005) y dar mayor valor agregado tanto a la organización como a sus clientes. La creciente importancia de la innovación tecnológica, crucial para la creación y sostenimiento de ventajas competitivas (Essman, 2009), (Tidd, 2009) es debida en parte a la globalización de los mercados ya que la competencia ha presionado a las empresas e instituciones a innovar continuamente (Schilling, 2010); La tecnología dada su naturaleza sistémica (Arthur, 2009), por su parte ha jugado un papel importante en la productividad y competitividad de empresas, regiones y países, conocer los perfiles o diagnósticos tecnológicos, de innovación y de estrategia de organizaciones y sus correspondientes brechas tecnológicas es indispensable para elaborar y generar objetivos, estrategias y proyectos.

Tradicionalmente, las Universidades han tenido como funciones básicas la formación, la generación, la transmisión del conocimiento y la producción de servicios para la sociedad, y solo hasta hace poco tiempo han pensado en la innovación como un aspecto para mejorar la productividad y competitividad. La creación de oficinas de transferencia de tecnología dentro de las universidades ha sido el primer paso para lograr que sus investigaciones produzcan una innovación comercial, al igual que la publicación de resultados de investigación que se incorporan en esfuerzos de desarrollo de otras organizaciones (Schilling, 2010).

Para que una Universidad sea considerada como innovadora, debe cumplir con algunas condiciones que hacen énfasis en las características propias de la institución, en las actividades que se llevan a cabo dentro de ella, la manera como se financia y los resultados que se obtienen a través de sus grupos y centros de investigación. Cabe resaltar los modelos de universidades innovadoras como es el caso de la Universidad de Harvard, que mantiene un sistema académico en el que hay una relación con el sector empresarial pero siempre a partir de las líneas estratégicas marcadas por la política de la universidad, y el modelo de la Universidad de Stanford en el que la institución está alineada con el sector empresarial.
Hablar de innovación en las instituciones de educación superior $y$ en sus grupos y centros de investigación es un tema que ha tomado mucha fuerza actualmente, ya que ésta actividad permite mejorar el direccionamiento estratégico en cuanto a productos/servicios y procesos pertinentes e innovadores.

Colombia desde la década de los 90 tiene establecido una política de apoyo y fomento para la consolidación de los grupos y centros de investigación lo que ha permitido una maduración constantes de algunas líneas de investigación y la visibilidad de las mismas a nivel internacional (Colciencias, 2008). Actualmente los grupos de investigación son la principal fuente de investigación e innovación al interior de las Instituciones de Educación Superior Colombiana y cabe destacar que en los últimos años han avanzado progresivamente en la consolidación de sus capacidades investigativas, sin embargo, se puede decir que estos presentan deficiencias en la integración a los sistemas de innovación ( Lundvall, 2009), lo cual conlleva a pensar que establecen una estrategia genérica basada exclusivamente en la Gestión de Investigación y Desarrollo y desconocen aspectos de suma importancia para generar innovación en productos y servicios, como es el caso de formas premeditadas de colaboración, mecanismos de propiedad intelectual y la falta de procedimientos para medir el impacto de sus innovaciones y su ciclo de innovación.

\section{Metodología}

La primera parte de la metodología utilizada para realizar el diagnóstico de estrategia de innovación de los grupos de investigación de la Universidad Pontificia Bolivariana fue el diseño de una encuesta, la cual se fundamentó en diferentes herramientas de diagnóstico como son: metodología MGT (Zartha, 2008), diagnóstico de innovación de Albacete, diagnóstico de capacidades de innovación de Cataluña y la metodología de estrategia de innovación (Schilling, 2010). La encuesta o herramienta de diagnóstico se dividió en 3 secciones las cuales se presentan a continuación:

Sección I. Datos Generales: Registro de los datos descriptivos del grupo de investigación, responsables y fechas de diligenciamiento. 
Sección 2. Innovación: Está orientada a determinar el estado actual de la estrategia de innovación y de los aspectos que fundamentan el proceso de la innovación. Entre estos aspectos se encuentran: la indagación sobre la existencia de un direccionamiento estratégico que recoja aspectos como la existencia de un portafolio de productos y servicios, el estado actual de los procesos de desarrollo de la innovación en el grupo, la identificación de mercado objetivo y la identificación de la posición del grupo en relación a la generación de nuevas ideas, la inventiva y la creatividad. Por otro lado en esta sección se indaga también sobre algunos aspectos fundamentales de la formulación y la implementación de la estrategia de innovación. En la formulación de la estrategia se pregunta sobre la cantidad de proyectos de innovación derivativos, de plataforma, de rompimiento y de avanzada según la clasificación propuesta por Schilling en su libro dirección estratégica de la innovación tecnológica (ver figura I), además se consulta sobre sus formas o estrategias de colaboración y los mecanismos de protección de la innovación (Tidd, 2009) que han implementado. En la implementación de la estrategia se generaron preguntas para poder indagar cuales herramientas o metodologías utilizan para mejorar el desarrollo de sus nuevos productos, procesos y servicios, como por ejemplo State Gate (Cooper, 2009), el análisis de ciclo de vida entre otras y por último se les preguntó sobre cómo estaba organizado su equipo de trabajo teniendo en cuenta la siguientes formas o estructuras: equipos funcionales, ligeros, pesados y autónomos. (Burgelman, 2009).

\section{Mapa de Proyectos para los Grupos de Investigación}

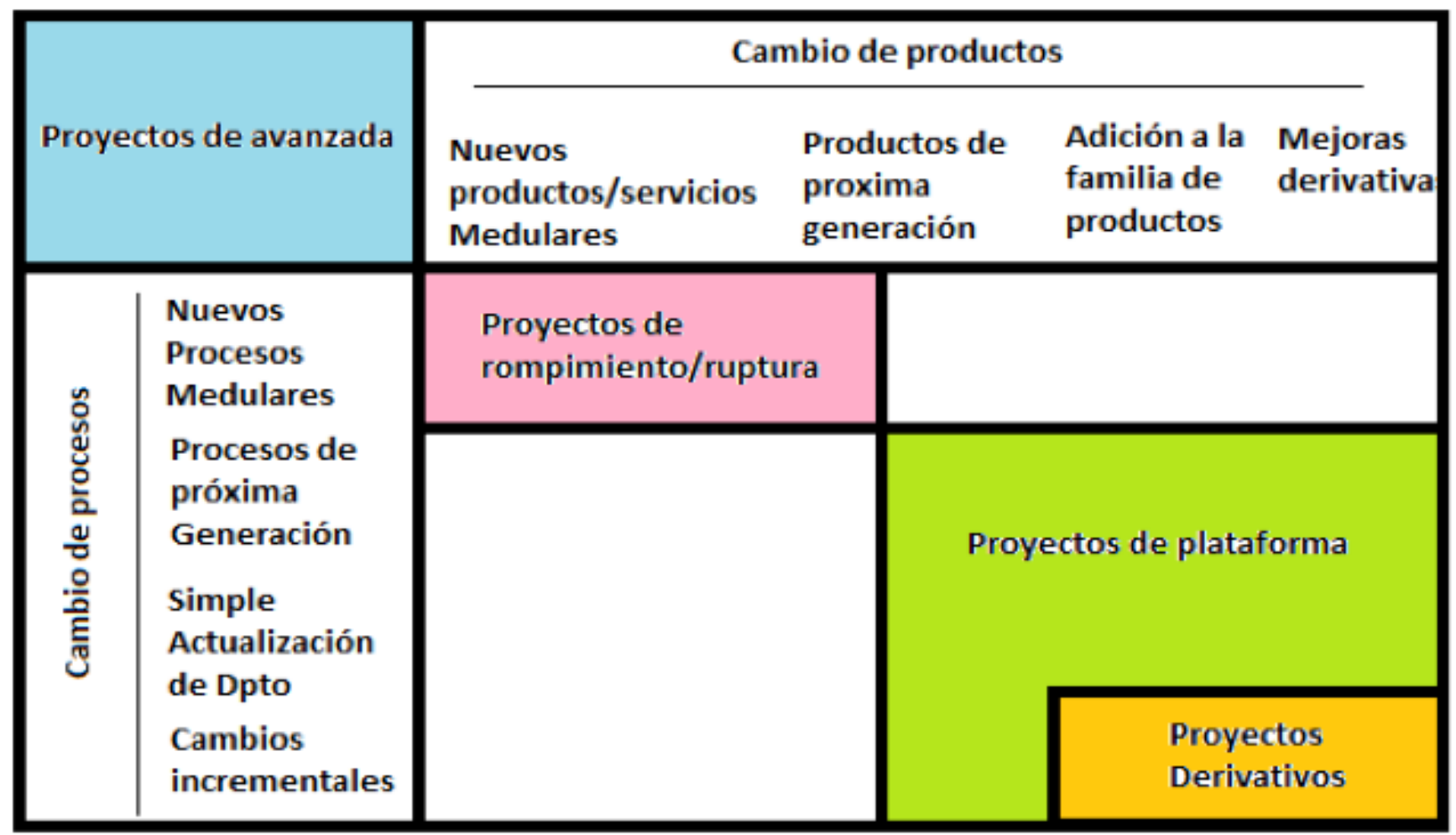

Figura I. Representación gráfica de los proyectos / Fuente: Schilling, Melissa 2010. 
Sección 3. Vigilancia Tecnológica: En esta sección se identifican las distintas maneras en que los grupos de investigación detectan y analizan la información que sirve de apoyo para generar la innovación (Sánchez y Palop, 2002).
Siguiendo con el desarrollo de la investigación la herramienta fue aplicada a 52 grupos de investigación los cuales se clasifican en tres áreas de conocimientos: Ciencias de la Salud, Ingenierías, Arquitectura y Diseño y Ciencias humanas y Sociales, luego de esto se realizó un análisis estadístico y se generaron conclusiones generales sobre el estado actual de los grupos de investigación.

\begin{tabular}{|c|c|c|c|}
\hline Area & Total Grupos & $\begin{array}{c}\text { Grupos } \\
\text { encuestados }\end{array}$ & $\begin{array}{c}\text { Porcentaje de } \\
\text { Grupos E ncuestados }\end{array}$ \\
\hline Ciencias de la Salud & 14 & 12 & $86 \%$ \\
\hline $\begin{array}{c}\text { Ciencias Humanas y } \\
\text { Sociales }\end{array}$ & 22 & 22 & $100 \%$ \\
\hline $\begin{array}{c}\text { Ingenier'as, Arquitectura } \\
\text { y Dise- 0 }\end{array}$ & 19 & 18 & $95 \%$ \\
\hline
\end{tabular}

Tabla I. Total Grupos de investigación encuestados por áreas de conocimiento / Fuente: Los autores

\section{Resultados}

A continuación se presentan los resultados obtenidos por sección, luego de realizar un análisis estadístico en donde se relacionan algunos elementos de la herramienta diagnóstico, se hacen observaciones generales y en ocasiones particulares para cada una de las áreas de conocimiento.

\section{I Sección Innovación:}

\section{I.I Direccionamiento estratégico de los grupos de investigación}

Dentro del diagnóstico realizado al interior de los grupos de investigación de la Universidad elegida se encuentra que en el eje de direccionamiento estratégico, la mayoría de los grupos de investigación a pesar de tener identificados los clientes no todos tienen definido un portafolio de productos y servicios, aunque cabe mencionar que el área de ingeniería evidencia un ingreso a nuevos mercados en los últimos tres años y el mayor porcentaje de sus grupos de investigaciónpresentaun portafolio deproductosyservicios.

En el área de la salud se encuentra que sólo la mitad de los grupos de I+D poseen un portafolio de productos y servicios definido. Es necesario que aquellos que no lo poseen inicien por construir su portafolio a partir de sus capacidades. 


\begin{tabular}{|c|c|c|c|c|}
\hline $\begin{array}{c}\text { ÈREA DEL } \\
\text { CONOCIMIENTO }\end{array}$ & ELEMENTOS Y PORCENTAJES & \multicolumn{3}{|c|}{ PORCENTAJES } \\
\hline \multirow{5}{*}{ Ciencias de la Salud } & \multirow{3}{*}{ Portafolio de Productos y Servicios definido } & SI & NO & NS/NR \\
\hline & & & & \\
\hline & & $50 \%$ & $50 \%$ & $0 \%$ \\
\hline & Identificaci-n de clientes & $67 \%$ & $25 \%$ & $8 \%$ \\
\hline & Acceso a nuevos mercados & $51 \%$ & $33 \%$ & $16 \%$ \\
\hline \multirow[t]{3}{*}{ Ciencias H umanas y Sociales } & Portafolio de Productos y Servicios definido: & $68 \%$ & $27 \%$. & $5 \%$ \\
\hline & Identificaci-n de clientes & $81 \%$ & $9 \%$ & $10 \%$ \\
\hline & Acceso a nuevos mercados & $63 \%$ & $27 \%$ & $10 \%$ \\
\hline \multirow{3}{*}{$\begin{array}{l}\text { Ingenier as, Arquitectura y } \\
\text { Dise- o }\end{array}$} & Portafolio de Productos y Servicios definido: & $82 \%$ & $18 \%$. & $0 \%$ \\
\hline & Identificaci-n de clientes: & $94 \%$ & $6 \%$ & $0 \%$ \\
\hline & A cceso a nuevos mercados & $76 \%$ & $24 \%$ & $0 \%$ \\
\hline
\end{tabular}

Tabla 2. Direccionamiento estratégico

\subsubsection{Selección de proyectos de innovación}

Entre las diferentes áreas del conocimiento, se rescata el área de Ciencias de la Salud la cual tiene un portafolio de proyectos con una mayor concentración en proyectos derivativos y de plataforma, y una menor concentración en los proyectos de rompimiento y avanzada (Schilling, 20I0).

Es importante anotar en general que dada la naturaleza de los proyectos en los grupos de investigación, el tipo de proyecto está condicionado por los requerimientos de los clientes, desde esta perspectiva los grupos están menos preocupados por un portafolio balanceado y más por satisfacer o cumplir la exigencias de los clientes y así permitir el sostenimiento del grupo. A continuación se resumen algunas características y porcentajes del mapa de proyectos, es decir, la distribución del portafolio de proyectos de los grupos de investigación. 


\begin{tabular}{|c|c|}
\hline ÇREA DE CONOCIMIENTO & CARACTER ÊSTICAS \\
\hline Ciencias de la Salud & $\begin{array}{l}\text { El } 83 \% \text { de los grupos tienen proyectos en las } 4 \\
\text { categor'as. Esta distribuci-n muestra cierto } \\
\text { balance donde ningom grupo tiene m } \neq s \text { del } 75 \% \text { de } \\
\text { sus proyectos en una sola categor'a y todos est } \neq n \\
\text { con menos del } 25 \% \text { en proyectos de avanzada. }\end{array}$ \\
\hline Ciencias H umanas y Sociales & $\begin{array}{l}\text { El } 90 \% \text { de los grupos tienen proyectos en las } 4 \\
\text { categor'as. Dentro de la distribuci-n se encontr- } \\
\text { un solo grupo con } \mathrm{m} \neq \mathrm{s} \text { del } 75 \% \text { en proyectos } \\
\text { derivativos, otro con } \mathrm{m} \neq \mathrm{s} \text { del } 25 \% \text { en proyectos de } \\
\text { avanzada y } 4 \text { grupos con } \mathrm{m} \neq \mathrm{s} \text { del } 50 \% \text { en } \\
\text { proyectos de rompimiento/ruptura. }\end{array}$ \\
\hline Ingenier'as, Arquitectura y Dise- 0 & 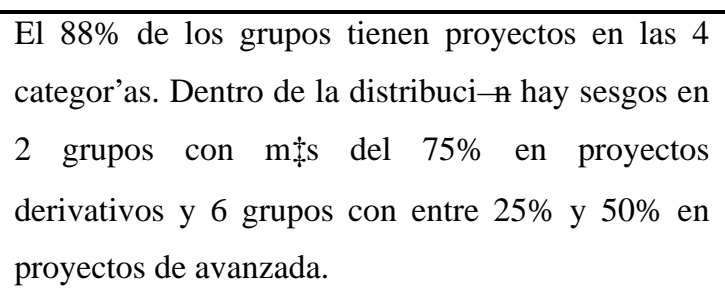 \\
\hline
\end{tabular}

Tabla 3. Selección de proyectos de $1+D+i$

\section{I.3 Estrategias de colaboración}

En general las estrategias de colaboración más utilizadas para el desarrollo de proyectos de investigación e innovación en los grupos de investigación son las alianzas estratégicas y la organización colectiva para la investigación
(Solleiro, 2009) las cuales se han convertido en un requisito para la presentación en convocatorias de investigación e innovación de orden gubernamental y no gubernamental. En la siguiente tabla se presenta el resumen del análisis estadístico realizado para esta parte.

\begin{tabular}{|l|l|}
\hline \multicolumn{1}{|c|}{$\begin{array}{c}\text { Ç REA DE } \\
\text { C ONOCIM IE NT O }\end{array}$} & \multicolumn{1}{c|}{ FOR M A S C O L A BORACIî N } \\
\hline Ciencias de la Salud & $\begin{array}{l}58 \% \text { de los grupos han participado en Alianzas EstratZgicas. 50\% en } \\
\text { Organizaci-n Colectiva para la Investigaci-n. 25\% en N inguna } \\
\text { forma de colaboraci-n. }\end{array}$ \\
\hline $\begin{array}{l}\text { Ciencias Humanas y } \\
\text { Sociales }\end{array}$ & $\begin{array}{l}68 \% \text { de los grupos han participado en A lianzas EstratŽgicas. 64\% en } \\
\text { Organizaci-n Colectiva para la Investigaci-n. 14\% en J oint-Venture } \\
23 \% \text { en Ninguna forma de colaboraci-n. }\end{array}$ \\
\hline $\begin{array}{l}\text { Ingenier'as, Arquitectura y } \\
\text { Dise- o }\end{array}$ & $\begin{array}{l}76 \% \text { de los grupos han participado en A lianzas EstratZgicas. 65\% en } \\
\text { Organizaci-n Colectiva para la Investigaci-n. 29\% en Outsourcing. } \\
24 \% \text { en Licenciamiento. }\end{array}$ \\
\hline
\end{tabular}

Tabla 4. Estrategias de colaboración 


\section{I.4 Protección a la innovación}

Se encuentra como elemento común que el mecanismo de protección de la innovación más utilizado por los grupos de investigación son las cláusulas de confidencialidad. Así mismo se evidencia que aproximadamente un cuarto de los grupos de investigación no utilizan ningún mecanismo de protección, lo cual implica que existe un alto riesgo desde la perspectiva de la protección de la información de aquellos proyectos que apunten a ser de innovación.
Otro punto interesante es que en todas las áreas del conocimiento existen proyectos de rompimiento y de avanzada en los cuales la mejor forma de protección es la utilización de patentes o modelos de utilidad, solo 10 grupos han utilizado estos mecanismos.

\begin{tabular}{|c|c|}
\hline ÇREA DE CONOCIMIENTO & CARACTER ÊSTICAS \\
\hline Ciencias de la Salud & $\begin{array}{c}67 \% \text { clfusulas confidencialidad } \\
25 \% \text { no ha utilizado ninguna proteccin } \\
4 \% \text { patente/modelo utilidad } \\
4 \% \text { marca/lema comercial }\end{array}$ \\
\hline Ciencias H umanas y Sociales & $\begin{array}{c}50 \% \text { cl } \neq \text { usulas confidencialidad } \\
32 \% \text { no ha utilizado ninguna proteccin } \\
12 \% \text { patente/modelo utilidad } \\
6 \% \text { marca/lema comercial }\end{array}$ \\
\hline Ingenier'as, Arquitectura y Dise- 0 & $\begin{array}{c}75 \% \text { clłusulas confidencialidad } \\
19 \% \text { no ha utilizado ninguna proteccin } \\
2 \% \text { disee i ndustrial/trazado circuitos } \\
3.5 \% \text { patente/modelo utilidad } \\
0.5 \% \text { marca/lema comercial }\end{array}$ \\
\hline
\end{tabular}

Tabla 5. Protección a la innovación

\section{I.5 Gestión del proceso de desarrollo de nuevos productos}

Un elemento común a la muestra de grupos es la definición de un líder para cada proyecto. La mayoría de los grupos de investigación no realizan procesos de stage-gate (de la idea a la validación comercial del producto) ni análisis de ciclo de vida del producto o servicio (indicador de nivel de aceptación del mercado). Esto quiere decir que aunque se tiene un líder asociado, al no poseer herramientas ade- cuadas de gestión se puede limitar la capacidad de innovar.

En la siguiente tabla se muestra el resumen por área de conocimientos, es importante aclarar que las respuestas no son mutuamente excluyentes, es decir, los grupos pueden utilizar varias de las características mencionadas, por lo que se pueden presentar valores totales superiores al $100 \%$. 


\begin{tabular}{|c|c|}
\hline ç REA DE CONOCIMIENTO & CARACTER R̂STICAS \\
\hline Ciencias de la Salud & $\begin{array}{c}92 \% \text { I'der por proyecto } \\
8 \% \text { desarrollo en paralelo } \\
0 \% \text { stage gate } \\
16 \% \text { Antlisis ciclo de vida producto }\end{array}$ \\
\hline Ciencias H umanas y Sociales & $\begin{array}{c}64 \% \text { l'der por proyecto } \\
41 \% \text { dllo en paralelo } \\
18 \% \text { stage gate } \\
14 \% \text { anłlisis ciclo de vida producto }\end{array}$ \\
\hline Ingenier'as, A rquitectura y Dise- 0 & $\begin{array}{c}63 \% \text { I'der por proyecto } \\
63 \% \text { dllo en paralelo } \\
11 \% \text { stage gate } \\
16 \% \text { anłlisis ciclo de vida producto }\end{array}$ \\
\hline
\end{tabular}

Tabla 6. Proceso de desarrollo de nuevos productos

2.I.6 Gestión de los equipos de desarrollo de nuevos productos
Se encuentra que la mayoría de los grupos de investigación tienen una estructura funcional o de peso liviano.

\begin{tabular}{|c|c|}
\hline Ç REA DE C ONOC IM IE NTO & CARACTE RêST ICAS \\
\hline Ciencias de la Salud & $50 \%$ funcional \\
& $17 \%$ liviano \\
& $8 \%$ pesado \\
Ciencias H umanas y Sociales & $8 \%$ ninguna estructura \\
\hline & $9 \%$ funcional \\
& $41 \%$ liviano \\
& $23 \%$ pesado \\
Ingenier'as, Arquitectura y Dise- & $13 \%$ ninguna estructura \\
& $24 \%$ funcional \\
& $29 \%$ liviano \\
& $27 \%$ pesado \\
& $11 \%$ ninguna estructura \\
\hline
\end{tabular}

Tabla 7. Gestión de equipos de desarrollo 


\section{I.2. Sección 3. Vigilancia Tecnológica:}

Se encontró que más de la mitad de los grupos analizados no realizan vigilancia sobre temas específicos. Además no cuentan con personas dedicadas a realizar vigilancia tecnológica. Este hecho per se no indica una falencia de los grupos, simplemente evidencia la necesidad de tener una unidad de apoyo en vigilancia tecnológica que sea transversal a los mismos.

Existe una aparente contradicción entre la captura de información de los hábitos de consumo de los clientes contra la captura de información de las tendencias del entorno. Por un lado aparece que menos de la mitad de los grupos de investigación captan información referente a los hábitos de consumo de los clientes, mientras que, por el otro más de tres cuartas partes de ellos declaran captar información de las tendencias del entorno.

En cuanto a la información, se puede decir que en general más de tres cuartas partes de los grupos tienen la información almacenada y disponible en medios electrónicos. Sin embargo en el área de Ciencias de la Salud ningún grupo tiene formatos estándar de recolección de información y en las otras dos áreas del conocimiento sólo II grupos declararon tenerlo. Ante estos hechos queda la duda sobre si efectivamente se están consultando estas bases de datos antes y durante el desarrollo de los proyectos y, si la forma en la cual se encuentra la información la hace realmente útil.

\begin{tabular}{|c|c|c|c|}
\hline $\begin{array}{c}\text { Ç REA DEL } \\
\text { CONOCIMIENTO / } \\
\text { êTEM }\end{array}$ & $\begin{array}{l}\text { CIENCIASDE LA } \\
\text { SALUD }\end{array}$ & $\begin{array}{c}\text { CIENCIAS HUMANAS } \\
\text { Y SOCIALES }\end{array}$ & $\begin{array}{c}\text { INGENIER } A \hat{A} \text {, } \\
\text { ARQUITECTURA Y DISE „O }\end{array}$ \\
\hline $\begin{array}{l}\text { Vigilancia de Temas } \\
\text { espec'ficos }\end{array}$ & $\begin{array}{c}\text { Si } 25 \% \text {. No } 67 \% \text {. NS/NR } \\
8 \% .\end{array}$ & $\begin{array}{c}\text { Si } 45 \% \text {. No } 50 \% \text {. NS/NR } \\
5 \%\end{array}$ & Si $25 \%$. No 67\%. NS/NR 8\%. \\
\hline $\begin{array}{l}\text { Personas dedicadas a } \\
\text { vigilancia }\end{array}$ & $\begin{array}{c}\text { Si } 8 \% . \text { No } 84 \% \text {. NS/NR } \\
8 \% .\end{array}$ & $\begin{array}{c}\text { Si } 36 \% . \text { No } 59 \% \text {. NS/NR } \\
5 \% .\end{array}$ & Si $23 \%$. No 71\%. NS/NR 6\%. \\
\hline $\begin{array}{l}\text { Vigilancia de } \mathrm{H} \ddagger \text { bitos de } \\
\text { consumo }\end{array}$ & $\begin{array}{c}\text { Si } 23 \% \text {. No } 71 \% \text {. NS/NR } \\
6 \% .\end{array}$ & $\begin{array}{c}\text { Si } 55 \% \text {. No } 36 \% \text {. NS/NR } \\
9 \% .\end{array}$ & Si 35\%. No 59\%. NS/NR 6\%. \\
\hline $\begin{array}{l}\text { Vigilancia de Tendencias } \\
\text { del entorno }\end{array}$ & $\begin{array}{c}\text { Si } 75 \% \text {. No } 17 \% \text {. NS/NR } \\
8 \% .\end{array}$ & $\begin{array}{c}\text { Si } 77 \% \text {. No } 14 \% \text {. NS/NR } \\
9 \% .\end{array}$ & Si $82 \%$. No $18 \%$. NS/NR 0\%. \\
\hline $\begin{array}{l}\text { Disponibilidad de la } \\
\text { informacia }\end{array}$ & $\begin{array}{c}\text { Si } 75 \% . \text { No } 17 \% \text {. NS/NR } \\
8 \% .\end{array}$ & $\begin{array}{c}\text { Si } 68 \% \text {. No } 27 \% \text {. NS/NR } \\
5 \% .\end{array}$ & Si $88 \%$. No $12 \%$. NS/NR 0\%. \\
\hline $\begin{array}{l}\text { Formatos estłndar para } \\
\text { vigilancia. }\end{array}$ & $\begin{array}{c}\text { Si } 0 \% . \text { No } 92 \% . \mathrm{NS} / \mathrm{NR} \\
8 \% .\end{array}$ & $\begin{array}{c}\text { Si } 27 \% . \text { No } 64 \% \text {. NS/NR } \\
9 \% .\end{array}$ & Si $25 \%$. No $65 \%$. NS/NR $10 \%$. \\
\hline
\end{tabular}

Tabla 8. Vigilancia Tecnológica

ISSN: 07I 8-2724. (http://www.jotmi.org) 


\section{Conclusiones}

En el presente trabajo se realizó un análisis de la información recopilada de 52 grupos de investigación que fue el resultado de aplicar una metodología construida a partir de herramientas sobre innovación y estrategia. Todo esto permitió concluir algunos aspectos generales como son:

- Se estudiaron 52 grupos de investigación en una Universidad de Medellín que se clasificaron en tres áreas de conocimientos: Ciencias de la Salud, Ingenierías, Arquitectura y Diseño y Ciencias humanas y Sociales.

- La mayoría de los grupos de investigación a pesar de tener identificados los clientes no todos tienen definido un portafolio de productos y servicios.

- El $88 \%$ de los grupos de Ingenierías, Arquitectura y Diseño tienen proyectos en las 4 categorías. Dentro de la distribución hay sesgos en 2 grupos con más del $75 \%$ en proyectos derivativos y 6 grupos con entre $25 \%$ y $50 \%$ en proyectos de avanzada.

- El 76\% de los grupos de Ingenierías, Arquitectura y Diseño han participado en Alianzas Estratégicas. 65\% en Organización Colectiva para la Investigación. $29 \%$ en Outsourcing. 24\% en Licenciamiento.

- El $67 \%$ de Ciencias de la Salud, el 50\% de Ciencias Humanas y sociales y el $50 \%$ e Ingenierías, Arquitectura y Diseño no realizan vigilancia tecnológica en temas específicos, lo cual representa un alto porcentaje.

- La mayoría de los grupos de investigación no realizan procesos de Stage-gate (de la idea a la validación comercial del producto).

- Se encuentra como elemento común que el mecanismo de protección de la innovación más utilizado por los grupos de investigación son las cláusulas de confidencialidad. Así mismo se evidencia que aproximadamente un cuarto de los grupos de investigación no utilizan ningún mecanismo de protección, lo cual implica que existe un alto riesgo desde la perspectiva de la protección de la información de aquellos proyectos que apunten a ser de innovación.

- Se encuentra que la mayoría de los grupos de investigación tienen una estructura funcional o de peso liviano.
- Los grupos de investigación no cuentan con una estrategia de innovación definida que pueda ser aplicada en sus proyectos y garantizar en un mayor grado de certeza el acercarce a la consecución de objetivos que puedan ser catalogados como innovadores, así sea en un menor grado.

- Este trabajo ofrece la base fundamental para la formulación e implementación de una estrategia de innovación en los grupos de investigación que permitirá generar una cultura de innovación en cuanto a productos, servicios, procesos y mercados así como mejorar su competitividad.

\section{Referencias}

ARTHUR, B (2009). The Nature of Technology: What It Is and How It Evolves. New York: Free Press.

BURGELMAN, A. 2009. Strategic management, Integrating Technology and Strategy: A general Management Perspective. Mc Graw Hill.Vol 5. no. Cap I.p. 4.

CENTRO EUROPEO DE EMPRESAS E INNOVACIÓN. Autodiagnóstico de la innovación. [En línea]. Albacete: El Autor, s.f. Disponible en: <http://www.ceeialbacete.com/ corps/ceeialbacete/url/autodiagnostico/autodiagnostico.

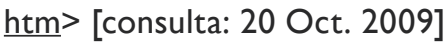

COLCIENCIAS. Colombia construye y siembra futuro. Política Nacional de fomento a la investigación y la innovación. Bogotá: El Autor, 2008.

COOPER, R. and Edgett, S. Lean, Rapid and Profitable New Product Development. USA: BookSurge Publishing. 2009

ESSMAN, H, Preez N 2009. Poceedings of Word Academy of Science, Engineering and TEchnology.An Innovation Capability Maturity Model-Development and inicial application.Vol 4 I p.435.

Instituto Catalán de Tecnología, Test de Innovación Empresarial ICT. <Disponible en: http://www.cea.es//UPLOAD/ INNOVACION/RECURSOS/INNO 9I_AI4\%20Test $\% 20$ de\%20innovacion\%20empresarial.pdf> [Consulta noviembre 5 de 2009] 
LUNDVALL, B., Vang, J., Joseph, K., Chaminade, C. (2009). Bridging Innovation System Research and Development Studies: challenges and research opportunities. 7th Globelics CConference, Senegal.

MARTINEZ, A (2009). Innovación y Competitividad en la sociedad y el Conocimiento. Ed. plaza Valdez. México

R, COOPER y E.J, Kleinschmidt, New product processes at leading industrial firms, industrial- marketing-management 20, n. 2 (1991), pp I37-48); y R.G. Cooper, Doing it right, ivey business journal $64, n^{\circ} 6$ ( 2000). Pp 54-6I

ROBLEDO VELÁSQUEZ, Jorge. Introducción a la Gestión Tecnológica. Medellín: Universidad Nacional de Colombia. Facultad de Minas. Escuela de Ingeniería de la Organización, 2005. 81 p.

SÁNCHEZ, J., \& Palop, F. (2002). Herramientas de Software para la práctica de la Inteligencia Competitiva en la empresa. Valencia: Triz XXI.

SOLLEIRO, L (2009).Gestión del conocimiento y centros de investigación y desarrollo de México, Brasil y Chile. Facultad latinoamericana de Ciencias Sociales.México.

SCHILLING, M. (2010). Strategic management of technological innovation. Editorial: MCGraw - Hill. New York.

TIDD, J. 2009. Managing Innovation, Integrating Technological, Market and Organizational Change. Vol. 4 no. Cap I, p.I7.

ZARTHA. S, W. 2008. Modelo Tecnológicos por Proyectos. Caso de aplicación. Universidad Pontificia Bolivariana Seccional Medellín. Vol.I p 19. 
J. Technol. Manag. Innov. 20II,Volume 6, Issue 3 